\title{
Design and Development of Smart Helmet
}

\author{
Dr.G RajivSureshKumar ${ }^{1}$, A. Priyadharshini ${ }^{2}$, B. Firoz $^{3}$ \\ Professor, JCT College of Engineering and Technology, Coimbatore, TamilNadu, India ${ }^{1}$ \\ IVCSE, JCT College of Engineering and Technology, Coimbatore, TamilNadu, India ${ }^{2,3}$
}

\begin{abstract}
Now a day, the number of two wheelers in India has drastically increased to a great extent. Due to which automobile users are undergoing numerous road accidents thereby causing death. The first and the foremost precaution to avoid Death is to protect our head, therefore we suggest this smart helmet using IOT. Even though people have helmet they are not using it properly. This system is a special idea which helps to promote the usage of helmet in order to make motorcycle driving safer than before. This system can help to know how safe the ride is or what can be made to make the ride safe. Using a force sensing device the helmet can be detected, with a gas detector consumption of alcohol can be known and many other components like SMTP, vibration detector and speed detectors are used.
\end{abstract}

Keywords: Force Sensing Device, Gas Detector, SMTP, Vibration Detector and Speed Detectors

\section{INTRODUCTION}

There is an increase in the usage of vehicles in the world which causes a large number of accidents day to day .There is no proper precaution taken to reduce the accidents. The first and foremost thing is to wear a helmet. By using a machine or device vehicles on road can be controlled manually. Saving a life is not as easy as possible to do but a helmet plays a vital role in this system. The helmet will have a force detecting sensor which detects whether we wear a helmet or not, if not the bike cannot be started. Then a gas detecting sensor is attached to it which indicates if the rider has consumed alcohol. A vibration detector is used to capture the vibration when the vehicle collide with the other and a mail is send to the corresponding user regarding the accident so that they can do the needful soon. Also a speed detector which alerts the police and the user with a buzzer

\section{METHODOLOGY}

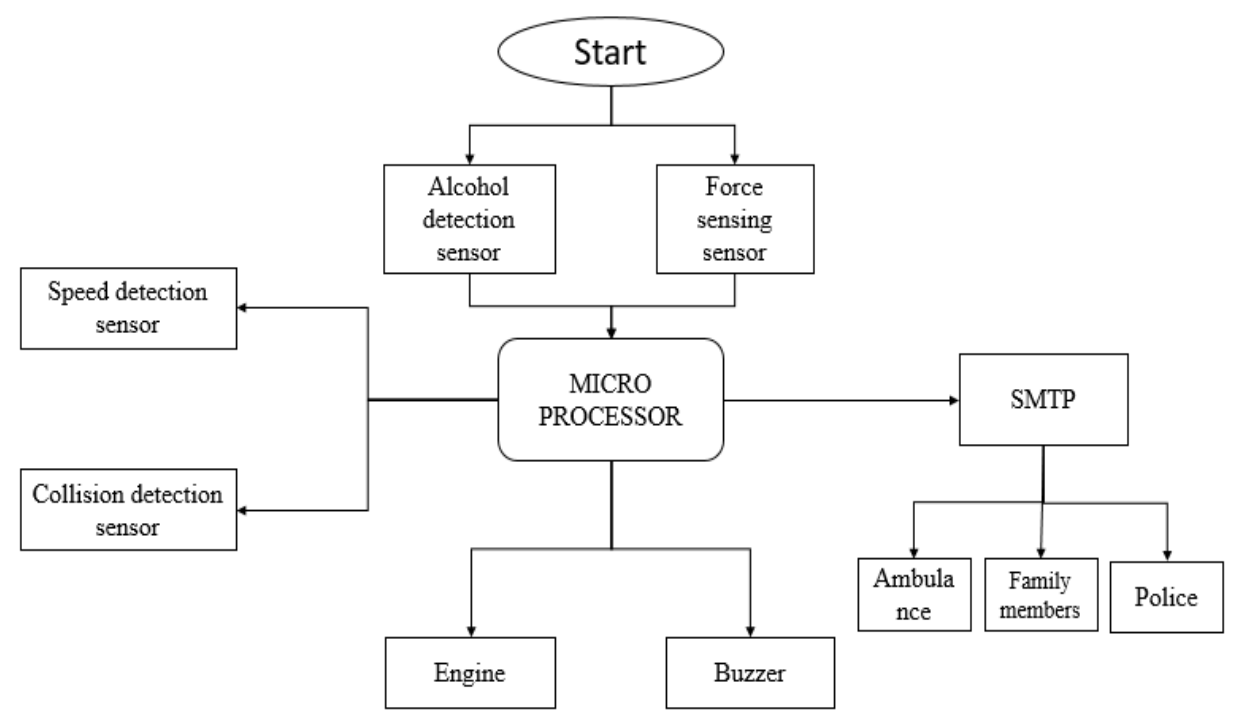

\section{1) ARDUINO:}

ARDUINO is both an equipment and a product that utilizes microcontroller units for building computerized gadgets that detects the articles in the genuine world. Arduino board can utilize an assortment of chip and controllers. In this we use ARDUINO to go about as an interface gadget with the GSM module so it gives the essential suggestion by sending the message to the relating individual when the individual has experienced an accident. Arduino programming enables us to compose the fundamental codings with the goal that the accompanying capacities can be translated. Arduino in every case is simple to learn and are additionally straightforward in its development and looks. 


\section{IJARCCE}

Vol. 8, Issue 2, February 2019

\section{2) SENSORS:}

sensors are advanced gadgets that are habitually used to recognize and react to electrical or optical signs. A Sensor changes over the physical parameter (for instance: temperature, pulse, moistness, speed, and so on.) into a flag which can be estimated electrically. Here we utilize four sorts of sensors like

\section{$>\quad$ Force sensing resistor \\ $>\quad$ Gas Detector \\ $>\quad$ Collision Detector or vibration detector \\ $>\quad$ Speed detector}

\section{3) SMTP:}

SMTP is developing as the a standout amongst the most significant administration in web today. The greater part of the web frameworks use SMTP as a technique to exchange mail starting with one client then onto the other. SMTP is a push convention and is utilized to send the mail though POP (post office convention) or IMAP (web message get to convention) are utilized to recover those send mails at the collector's side. SMTP is an application layer convention. The customer who needs to send the mail opens a TCP association with the SMTP server and after that sends the mail over the associate. When it tunes in for a TCP association from any customer, the SMTP procedure starts an association on that port. After effectively building up the TCP association the customer procedure sends the mail right away.

\section{PROPOSEDSYSTEMS}

The proposed system detects Alcohol consumption using the gas detector. If the speed exceeds it automatically sends an alert mail to the nearest police station and a buzzer is added to alert the rider if the speed exceeds. If accident occurs the system sends the information to the nearest hospital with the current location with SMTP.

\section{MODULEDESCRIPTION}

1) Force sensing resistor- Force sensing resistor is a special type of resistor which works when a pressure is applied on it .Both the electrically conducting and non-conducting particles are present on this sensing films which are formulated for improving mechanical properties. If we wear the helmet a pressure is applied on it which turns on the system. If the pressure is not sensed which means there is no helmet.

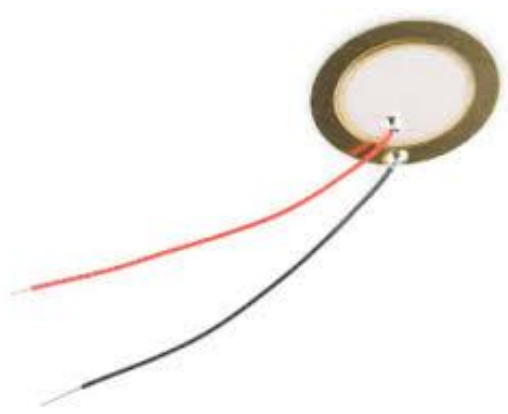

2) Gas Detector- Gas detector which detects the gas(alcohol)in the breath and indicates the rider has consumed alcohol and turns the system off so that they can not start the vehicle.

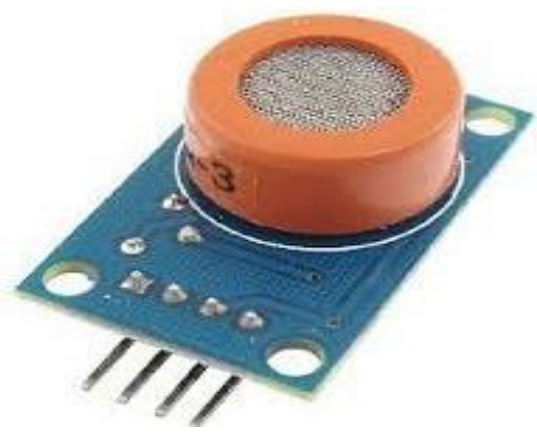




\section{IJARCCE}

Vol. 8, Issue 2, February 2019

3) Collision Detector or vibration detector- Collision detection or vibration sensor which knows the collision of vehicles and a mail is send to the hospital or the corresponding user to alert them with the accident using the Simple mail transfer protocol.

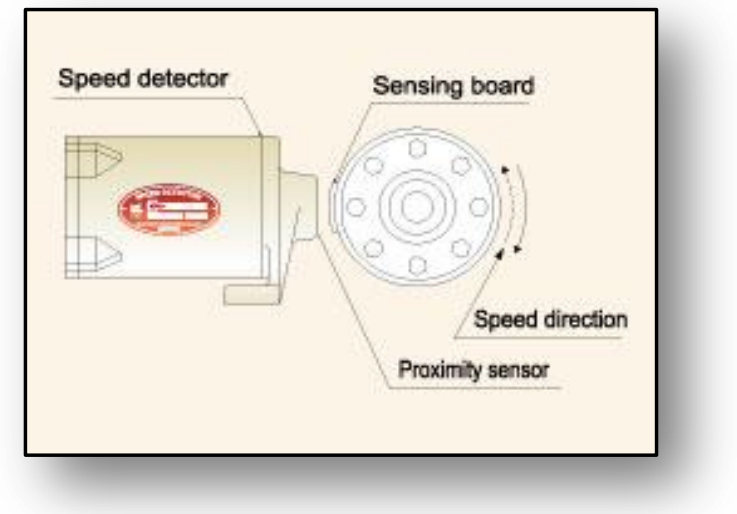

4) Speed detector-A Speed detector is used to detect the speed of the vehicle. If the speed exceeds it alerts the rider with a buzzer and sent the information to the police via E-mail.

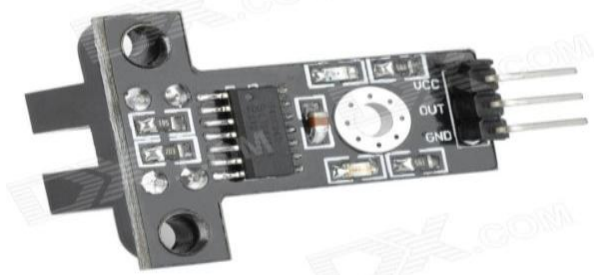

\section{CONCLUSION}

Road safety is an essential thing for all the people. According to the research road is the easiest and the most used mode of transport. To use these roads for safely every two wheelers should wear helmet which saves our valuable life and time. The above system will do the needful to the society. The police can also get the over speed riders.

\section{FUTURE SCOPE}

With this system It can prevent drink and drive. In emergency situation loss of lives can be prevented. Over speed can be detected by the police. Accident rates will be decreased. Buzzers can remind the riders about the over speed. The accidents rates will be decreased and more number of lives can be saved.

\section{REFERENCES}

[1] Amitava Das, Priti Das, Soumitra Goswami, "Smart Helmet For Indian Bike Riders", Proceedings of Eleventh IRF International Conference, 17th August 2014, Chennai, India, ISBN: 978-93-84209-47-6.

[2] K. Rambabu, B. Premalatha, C.Veeranjaneyulu, “An Optimal Driving System By Using Wireless Helmet”, International Journal of Science, Engineering and Technology Research (IJSETR) Volume 2, Issue 9, September 2013.

[3] Saadeddin, Kamal, Mamoun F. Abdel-Hafez, and Mohammad Amin Jarrah. "Low-cost, high-accuracy, state estimation for vehicle collision prevention system." Mechatronics and its Applications (ISMA), 2012 8th International Symposium on. IEEE, 2012.

[4] Hossain, A., et al., "A smartphone-assisted post-disaster victim localization method," IEEE 18th International Conference on High Performance Computing and Communications, pp. 1173-1179. IEEE 2016.

[5] Hyesun, L., et al., "An Internet of Things System Architecture for Aiding Firefighters in the Scene of Disaster," World Congress on Information Technology Applications and Services 2018 